\title{
Übergang in den Arbeitsmarkt nach einer Attestausbildung
}

\author{
Claudia Hofmann \& Kurt Häfeli
}

\section{Zusammenfassung}

Mit der Einführung der zweijährigen Grundbildung mit eidgenössischem Berufsattest (EBA) sollten die Arbeitsmarktfähigkeit der Absolventen und Absolventinnen und die Durchlässigkeit zu weiterführenden Ausbildungen im Vergleich mit der ehemaligen Anlehre verbessert werden. Die Hochschule für Heilpädagogik Zürich (HfH) führte dazu im Zeitraum 2006-2012 eine Längsschnittstudie mit drei Messzeitpunkten in vier Branchen (Gastronomie, Detailhandel, Hauswirtschaft und Schreinerei) durch. Zentral war dabei u.a. die Frage, ob sich das Ausbildungsgefäss sowohl für Lernende aus Regelklassen wie aus Sonderklassen eignet und wie sich die jeweiligen beruflichen Laufbahnen nach dem Abschluss weiterentwickeln. Es zeigt sich, dass die Lernenden aus Sonderklassen/-schulen etwas zufriedener mit ihrer Ausbildungssituation sind und sich weder im schulischen noch im betrieblichen Umfeld zu stark belastet fühlen. Sie haben zwar einen erschwerten Einstieg direkt nach Abschluss (höhere Erwerbslosigkeit, häufiger Temporäranstellungen), ihre Situation verbessert sich jedoch zum dritten Befragungszeitpunkt hin. Neben der schulischen Herkunft sind weitere Faktoren für die berufliche Integration von Bedeutung, wie z.B. die psychische Gesundheit, die Beurteilung der Lehrbetriebssituation, Werthaltungen in Bezug auf die Arbeit, schulische Leistungen und die schulische Belastung. 


\section{Abstract}

With the introduction of the two-year apprenticeship with Federal VET Certificate, the labor market ability of the graduates and the permeability to continuing education ought to be improved. In the period of time from 2006 to 2012, the University of Applied Science in Special Needs Education Zurich $(\mathrm{HfH})$ carried out a longitudinal study with three measuring times in four economic sectors (gastronomy, retail sales, home economics, joinery). One important question was if the new type of apprenticeship is suitable for both the learners from regular classes and from special needs classes and how the respective careers will develop after leaving school. The results show that the learners from special needs classes are somewhat more satisfied with their training situation and do not feel too much burdened whether in their school nor in their working environment. They do, however, have a more difficult start immediately after leaving school (higher unemployment, more frequently temporary jobs), but their situation is improving towards the third questioning point of time. Besides the school background, further factors are important for the vocational integration such as for instance mental health, the situation in the training companies, values with regard to work, and performances and stress at VET-school.

\section{Résumé}

L'introduction de la formation professionnelle initiale de deux ans avec attestation fédérale (AFP) visait à améliorer l'employabilité des diplômés et l'accès aux formations continues comparé à l'ancienne formation élémentaire. Entre 2006 et 2012, la Haute Ecole pour la pédagogie curative à Zurich (HfH) a effectué une étude longitudinale avec trois mesures dans quatre branches (gastronomie, commerce de détail, économie domestique et menuiserie). Une des questions centrales, c'était si le type de formation est approprié aux apprenants tant de classes régulières que de classes spécialisées ainsi que la question comment les orientations professionnelles des deux groups se développent après le diplôme. On constate que les apprenants des classes/écoles spécialisées sont un peu plus satisfaits avec leur situation de formation et qu'ils ne se sentent surchargé ni dans le contexte scolaire ni dans le contexte de l'entreprise. Immédiatement après le diplôme, leur entrée est plus difficile (plus de chômage, plus de contrats à durée déterminée), mais leur situation s'améliore vers la troisième mesure. Outre la provenance scolaire, d'autres facteurs influencent 
l'intégration professionnelle, comme p. ex. la santé psychique, l'évaluation de la situation dans l'entreprise, les valeurs par rapport au travail, les résultats scolaires et la charge scolaire.

\section{Ausgangssituation und Fragestellungen der HfH-Längsschnittstudie}

Der Übergang von der Schule in die Ausbildung und in den Arbeitsmarkt ist eine der wichtigsten Entwicklungsaufgaben, die sich in der Adoleszenz und im jungen Erwachsenenalter stellen (Grob \& Jaschinski, 2003). Für die betroffenen Jugendlichen bedeutet dieser Kontextwechsel einerseits eine Stimulation und Bereicherung, andererseits müssen erhebliche Anpassungsleistungen an die neue Situation vollbracht werden (Elfering, Semmer, Tschan, Kälin, \& Bucher, 2007; Neuenschwander, Gerber, Frank, \& Rottermann, 2012). Trotz einer breiten Palette von Ausbildungsmöglichkeiten bleibt in der Schweiz fast jeder zehnte junge Erwachsene ohne anerkannte Ausbildung auf der Sekundarstufe II (OECD, 2013). Das bildungspolitische Ziel von 95\% erfolgreichen Abschlüssen auf Sekundarstufe II bis 2015 ist somit noch nicht erreicht. Besonders gefährdet sind in dieser Phase Jugendliche mit einer Behinderung oder Beeinträchtigung und Jugendliche aus problematischen familiären Verhältnissen und mit Migrationshintergrund (Felkendorf \& Lischer, 2005; Häfeli \& Schellenberg, 2009; Heinz, 2002; Hupka, Sacchi, \& Stalder, 2006; Neuenschwander \& Grunder, 2010; OECD, 2000). Als Risikogruppe gelten auch Jugendliche aus einem Schultyp mit niedrigeren Anforderungen, z.B. aus Sonderklassen oder Sonderschulen (Eckhart \& Sahli Lozano, 2014; Gyseler, 2008).

Vor rund zehn Jahren (2004) trat ein neues Berufsbildungsgesetz in Kraft, das mit der schrittweisen Einführung der Grundbildung mit Eidgenössischem Berufsattest (EBA) für diese Gruppen von Jugendlichen etliche Neuerungen mit sich brachte (siehe Kap. 1 Thematische Einführung und Überblick). Diese zweijährige Ausbildung „dient der Vermittlung von Qualifikationen zur Ausübung eines Berufs mit einfacheren Anforderungen“ (www.sddb.ch) und wird mit einem standardisierten Qualifikationsverfahren abgeschlossen. Ziel ist es, die Arbeitsmarktfähigkeit und Durchlässigkeit zu weiterführenden Ausbildungen (speziell zum Eidgenössischen Fähigkeitszeugnis EFZ) zu verbessern. Lernende, deren Ausbildungserfolg gefährdet ist, werden zudem durch eine „fachkundige individuelle Begleitung" (FiB) gestützt. Die Grundbildungen mit EBA haben sich inzwischen mit 54 in Kraft getretenen Verordnungen (Stand September 2014) und 
rund 11'000 Lernenden (1. und 2. Lehrjahr, Bundesamt für Statistik 2012/2013) zu einem bedeutsamen Ausbildungsgefäss entwickelt.

Von Beginn weg wurde die berufliche Grundbildung mit EBA aber auch kritisch beobachtet, da man befürchtete, leistungsschwächere Lernende könnten von den erhöhten Anforderungen im neuen Ausbildungsgefäss überfordert sein (Lischer, 2005). Vor diesem Hintergrund initiierte die Hochschule für Heilpädagogik Zürich zwei Längsschnittstudien in vier Branchen - Detailhandel/Gastronomie (2006-2010) und Hauswirtschaft/Schreinerei (2009-2012). Im folgenden Beitrag soll der Frage nachgegangen werden, wie sich die schulische Herkunft auf die Ausbildungssituation und die weitere berufliche Laufbahn auswirkt: Wie zufrieden sind Jugendliche aus Sonderklassen oder -schulen mit ihrer Ausbildungssituation und wie belastet fühlen sie sich in der Berufsfachschule und im Betrieb im Vergleich mit Jugendlichen, die vor Ausbildungsbeginn eine Regelklasse besucht haben? Wie unterscheidet sich die berufliche Laufbahn nach Ausbildungsabschluss zwischen diesen beiden Gruppen und welche Faktoren sind hier weiter relevant?

\section{Einflussfaktoren auf frühe berufliche Laufbahnen}

\subsection{Der Einfluss der schulischen Herkunft}

Das schweizerische Schulsystem ist im internationalen Vergleich nach wie vor eines der selektivsten und die Zuweisung zu Schultypen erweist sich an verschiedenen Schlüsselstellen der frühen beruflichen Laufbahn als bedeutsamer Faktor (Häfeli \& Schellenberg, 2009; Neuenschwander, 2014). Bereits in der obligatorischen Schulzeit stellen verschiedene Schultypen trotz programmierter Durchlässigkeit unterschiedliche Entwicklungsmilieus dar (Hupka-Brunner \& Wohlgemuth, 2014) und beeinflussen so u.a. Lernangebote, Selbstwirksamkeitserwartungen, Leistungsmotivation und berufliche Ambitionen (Pinquart, Juang, \& Silbereisen, 2003). Lernende mit besonderem Förderbedarf haben auch in einem integrativen Kontext ein vergleichsweise tieferes schulisches Fähigkeitsselbstkonzept (Venetz, Tarnutzer, Zurbriggen, \& Sempert, 2012).

Die Bedeutung des Schultyps bei der Lehrstellensuche ist demgegenüber umstritten: Auf der einen Seite wird argumentiert, dass für Betriebe nicht primär der Schultyp entscheidend ist, sondern oft wichtiger, ob eine Person motiviert ist und in eine bestimmte Funktion und ins Team passt (Imdorf, 2014; Neuenschwander, 2014). Andererseits fungiert der Schultyp für viele Betriebe als Leistungslabel, um abschätzen zu können, ob Bewerber/-innen den schulischen Teil der Ausbildung 
bestehen können (Hupka-Brunner \& Wohlgemuth, 2014; Imdorf, 2014). Damit lässt sich im Bewerbungsprozedere Zeit- und Aufwand sparen (Eckhart \& Sahli Lozano, 2014), was sich insbesondere für Grossbetriebe als relevant erwies (Moser, 2004). Besonders hoch sind dadurch die Hürden für Lernende aus Sonderklassen und Sonderschulen (Eckhart \& Sahli Lozano, 2014; Gyseler, 2008) und oft bleibt v.a. für Sonderschüler/-innen als Alternative nur eine Ausbildung im geschützten Rahmen (Fasching, 2013). Zudem finden sich Lernende aus Real- oder Sonderklassen häufiger in Ausbildungsberufen mit niedrigerem Anforderungsniveau (Hupka-Brunner \& Wohlgemuth, 2014) und nicht in ihrem Wunschberuf (Eckhart, Blanc, \& Sahli, 2010).

Auch die Ausbildungssituation wird von Lernenden aus Sonderklassen/-schulen kritischer beurteilt. Sie verfügen ausserdem über ein kleineres soziales Netz und ein niedrigeres Fähigkeitsselbstkonzept im Vergleich mit Lernenden aus Regelklassen (Eckhart \& Sahli Lozano, 2014). Besorgniserregend ist weiter der Befund, dass die Lehrabbruchquoten in Berufen mit tieferem Anforderungsniveau (z.B. EBA-Ausbildung) markant höher sind als in Berufen mit höherem Anforderungsniveau (Stalder \& Schmid, 2006; Stern, Marti, von Stokar, \& Ehrler, 2010) und der Schultyp auch die Wiedereinstiegschancen nach einer Lehrvertragsauflösung massgeblich beeinflusst (Schmid \& Stalder, 2007, 2008). Insgesamt ist das Risiko, ausbildungslos zu bleiben bei Lernenden aus Schultypen mit tieferen Anforderungen deutlich erhöht (Bertschy, Böni, \& Meyer, 2007; Scharenberg, Rudin, Müller, Meyer, \& Hupka-Brunner, 2014).

Inwieweit sich der vor der Ausbildung besuchte Schultyp darüber hinaus auf die Arbeitsmarktchancen auswirkt, ist noch wenig erforscht, wie auch insgesamt noch wenig bekannt ist über die Situation nach Abschluss einer zweijährigen Grundbildung mit EBA (Stern et al., 2010). In Analysen von TREE (Geier, Hupka-Brunner, \& Gaupp, 2013) erweisen sich Schulnoten und das Niveau der abgeschlossenen Ausbildung als wichtig für einen erfolgreichen Übergang an der zweiten Schwelle, während der Schultypus vor Ausbildungsbeginn seinen Einfluss in multivariaten Analysen oft verliert.

\subsection{Weitere Einflussfaktoren}

Die schulische Herkunft ist nur einer von vielen Faktoren, die bei den verschiedenen Teil-Übergängen von der Schule in die Arbeitswelt eine Rolle spielen (vgl. Neuenschwander, 2014). Ob und wie weit sich die schulische Herkunft auf die Laufbahn auswirkt, ist somit "variabel und kontextabhängig“ (Imdorf, 2014, S.43) und die berufliche Laufbahn ein Produkt individueller Biografien und Ent- 
scheidungen, institutioneller Selektion und weiterer Kontextfaktoren (Häfeli \& Schellenberg, 2009). Diskutiert werden im Folgenden der Einfluss (1) der sozialen Schicht und des Migrationshintergrunds, (2) der Ausbildungsbedingungen und (3) der individuellen Dispositionen.

(1) Trotz zahlreicher Studien ist die Ergebnislage zum Einfluss der sozialen Schicht auf die berufliche Laufbahn inkonsistent: Als erwiesen gilt, dass die soziale Herkunft sich bereits auf der Sekundarstufe I auf die Leistungen und die Vergabe von Übergangsempfehlungen auswirkt, somit in einem engen Zusammenhang mit dem Schultypus steht (z.B. Maaz \& Neumann, 2014) und als Folge die Positionierung und das Niveau der Ausbildung beeinflusst (Häfeli \& Schellenberg, 2009). Analysen der TREE-Längsschnittdaten zeigen weiter, dass ein tiefer sozioökonomischer Status das Risiko erhöht, ausbildungslos zu bleiben (Bertschy et al., 2007) und, dass sich ein hoher Sozialstatus der Eltern auf den erreichten Status im späteren Erwerbsberuf auswirkt (Gaupp, Geier, \& Hupka-Brunner, 2012). Demgegenüber erweist sich eine höhere soziale Schicht in anderen Studien als wenig bedeutsam (Opheim, 2007) oder verzögert sogar den Einstieg in den Arbeitsmarkt (Geier et al., 2013; Rüfenacht \& Neuenschwander, 2014)2014. Konsistenter sind dagegen die Ergebnisse zum Einfluss des Migrationshintergrunds: Migranten/innen haben mehr Mühe einen passenden Ausbildungsplatz zu finden (Imdorf, 2014), schätzen ihre Ausbildungssituation kritischer ein (Müller, 2009), haben ein erhöhtes Risiko, ohne nachobligatorischen Bildungsabschluss zu bleiben (Scharenberg et al., 2014), mehr Schwierigkeiten an der „Zweiten Schwelle“ (Geier et al., 2013) und schlechtere Aussichten auf dem Arbeitsmarkt (Seibert \& Solga, 2005).

(2) Bezüglich Ausbildungsbedingungen ist Unterstützung im Ausbildungsumfeld wichtig, v.a. für Jugendliche mit schulischen oder anderen Schwierigkeiten (Hofmann \& Schaub 2014). Sie beeinflusst erwiesenermassen u.a. die Kompetenzentwicklung in der Berufsfachschule (Neuenschwander, Frey und Gasser 2007), den Selbstwert (Linnehan, 2003) und die spätere Laufbahnzufriedenheit (Ng. et al 2005). Zum anderen tragen Autonomie und Vielfalt bei der Arbeit im Lehrbetrieb zu positiver Leistungseinschätzung, zu beruflicher Identifikation und Arbeitszufriedenheit bei (Humphrey, Nahrgang, \& Morgenson, 2007; Ng, Eby, Sorensen, \& Feldman, 2005), begünstigen berufliche Aspirationen (Hofmann, Stalder, Tschan, \& Häfeli, 2014) und wirken sich positiv auf die spätere berufliche Integration aus (Stalder, 2012).

(3) Auf der Ebene der individuellen Dispositionen erweisen sich z.B. Persönlichkeitsmerkmale wie Extraversion, proaktives Verhalten, Kontrollüberzeugung oder tiefe Werte bei Neurotizismus (Ng et al 2005) oder Selbstwert und Durchsetzungsvermögen (Neuenschwander, Frey, \& Gasser, 2007), Ausbildungsmotivation und beruflicher Zukunftsoptimismus (Lehmann \& Seeber, 2007; Neuenschwan- 
der et al., 2007) als relevant für die Arbeits- oder Ausbildungszufriedenheit. Weiter begünstigen erwartungsgemäss verschiedene kognitive Fähigkeiten (z.B. Lesekompetenz) oder Schulnoten die berufliche Laufbahn (Geier et al., 2013; Spiess Huldi, Häfeli, \& Rüesch, 2006; Stalder, Meyer, \& Hupka-Brunner, 2008). Dagegen erwiesen sich die Leistungen in Schule und Betrieb (unter Kontrolle anderer Faktoren wie Werte und Erwartungen) in einer anderen Studie (Neuenschwander, 2014) als nicht relevant für die Wahrscheinlichkeit einer Weiterbildung oder für die Wahl einer unstrukturierten Zwischenlösung.

\subsection{Subjektive und objektive Indikatoren eines erfolgreichen Übergangs}

Die erwähnten Forschungsergebnisse beziehen sich auf unterschiedliche „outcome"-Variablen und es stellt sich die Frage, was in unserem Zusammenhang ein erfolgreicher Übergang konkret bedeutet. „Geglückte Übergänge“, so HupkaBrunner und Wohlgemuth (2014), zeichnen sich durch eine Passung von institutionellen oder gesellschaftlichen Anforderungen und individuellen Kompetenzen bzw. Entwicklungspotentialen aus. Auch der Begriff „Arbeitsmarktfähigkeit“verweist auf eine Form von Passung und wird definiert als „relative Fähigkeit einer Person, unter Berücksichtigung der Interaktion zwischen ihren persönlichen Eigenschaften und dem Arbeitsmarkt eine Beschäftigung zu finden" (Raeder \& Grote, 2003, S. 9). Inwieweit diese Passung hergestellt werden kann, lässt sich an verschiedenen Indikatoren messen, wobei oft zwischen „subjektivem Laufbahnerfolg“ (z.B. Arbeitszufriedenheit) und „objektivem Laufbahnerfolg“" (z.B. Lohn) unterschieden wird (Ng et al., 2005).

Heslin (2005) betont darüber hinaus, dass die unterschiedlichen Kontexte mitberücksichtigt werden sollten: Für die Ausbildungszeit sind das Durchhalten und der erfolgreiche Abschluss sowie der Einstieg in eine adäquate Arbeitsstelle oder eine Weiterbildung (z.B. eidg. Fähigkeitszeugnis) wichtige objektive Indikatoren (vgl. Häfeli \& Schellenberg, 2009). Subjektive Indikatoren sind beispielsweise positive Leistungseinschätzungen in der Berufsfachschule und im Betrieb, die Ausbildungszufriedenheit und Verbundenheit mit dem Beruf (Hofmann \& Häfeli, 2012) oder die spätere Arbeitszufriedenheit. Wie die Metaanalyse von Ng et al (2005) zeigt, beeinflussen soziodemografische Faktoren und „Humankapital“ (z.B.Ausbildungs-/Arbeitserfahrungen) eher den objektiven Laufbahnerfolg, während betriebliche Unterstützung und stabile Persönlichkeitsmerkmale eher den subjektiven Laufbahnerfolg vorhersagen. Abbildung 1 fasst die für unseren Kontext relevanten Einflussfaktoren zusammen. 


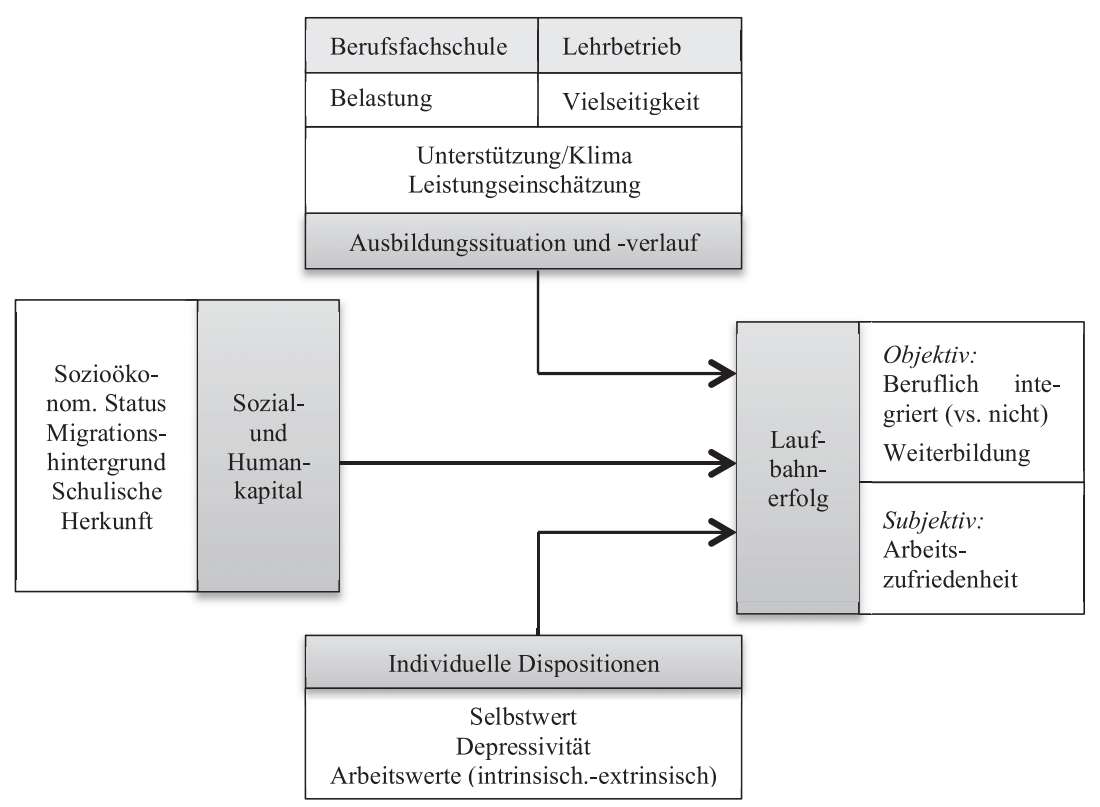

Abbildung 1 Einflussfaktoren auf die beruflichen Laufbahnen der EBA-Lernenden.

In unserem Kontext sind in Bezug auf den Einfluss der schulischen Herkunft verschiedene Szenarien denkbar: Jugendliche, welche die obligatorische Schulzeit in separierten Sonderklassen oder in einer Sonderschule absolvierten, sind im Vergleich mit Lernenden aus Regelklassen in der Ausbildung stärker gefordert und unter Umständen auch überfordert (vgl. Hofmann \& Kammermann 2008). Andererseits interpretieren sie die Ausbildung möglicherweise eher als Chance und sind motivierter als Jugendliche aus Regelklassen. Die zusätzliche Unterstützung während der Ausbildung („fachkundige individuelle Begleitung“) sollte zudem dazu beitragen, dass auch Lernende mit Schwierigkeiten die Ausbildung erfolgreich bewältigen und abschliessen können. Für die weitere Laufbahn ist zu vermuten, dass Absolventen/-innen unabhängig vom Schultyp dank der gesamtschweizerisch klar definierten Standards auf dem Arbeitsmarkt im Vergleich mit der früheren Anlehre bessere Karten haben, da künftige Arbeitgeber wissen, von welchen Kompetenzen sie bei diesen Jugendlichen ausgehen können. Im Arbeitsmarkt könnten somit die ungleichen Chancen aufgrund der früheren Separierung wieder ausgeglichen werden (vgl. auch Geier et al., 2013). In diesem Zu- 
sammenhang spielen allerdings auch die Akzeptanz und Bekanntheit des neuen Ausbildungsgefässes bei potentiellen Arbeitgebenden sowie die aktuelle regionale Arbeitsmarktsituation eine grosse Rolle.

Für die anderen Faktoren gehen wir auf der Basis der zitierten Forschungsliteratur davon aus, dass (1) soziodemographische Faktoren nur marginal wirken, weil bereits in früheren Selektionsprozessen, insbesondere bei der Lehrstellensuche entscheidender, (2) die Ausbildungsbedingungen einen zusätzlichen positiven Einfluss haben bzw. Belastung während der Ausbildung sich negativ auf die beruflichen Ambitionen auswirkt (wobei wir für die beiden Lernfelder unterschiedliche Faktoren vorschlagen, vgl. Abb. 1 und Kap. 2.2.) und (3) individuelle Dispositionen (Selbstwert, Depressivität) den jeweils erwarteten Effekt haben und auf den „subjektiven Laufbahnerfolg“ einwirken (vgl. Ng et al, 2000).

\section{$3 \quad$ Methodisches Vorgehen}

\subsection{Organisation der Befragungen, Erhebungsdesign und-instrumente}

Die erste Längsschnittstudie in den Branchen Gastronomie und Detailhandel (2006-2010) unter Einbezug der letzten Anlehrklassen wurde in Kooperation mit dem "Service de la Recherche en Éducation“ in Genf (SRED) durchgeführt und durch das SBFI mitfinanziert. Die Befragungen fanden in der Deutschschweiz, der französischsprachigen Schweiz und im Kanton Tessin statt. Die zweite Kohorte in den Branchen Hauswirtschaft und Schreinerei startete 2009 (bis 2012). Bei der Auswahl der Branchen wurden die damals wichtigen Branchen, gemessen an der Anzahl der Lehrverträge (gemäss Bundesamt für Statistik), berücksichtigt. Tabelle 1 zeigt die verschiedenen Erhebungszeitpunkte in ihrer zeitlichen Abfolge im Überblick, wobei nur die EBA-Lernenden dargestellt sind, da nur sie in den folgenden Analysen berücksichtigt wurden: 
Tabelle 1 Erhebungsdesign im Überblick und Stichprobengrössen.

\begin{tabular}{|c|c|c|c|c|c|c|}
\hline Befragte Personengruppen & 2007 & 2008 & 2009 & 2010 & 2011 & 2012 \\
\hline $\begin{array}{l}\text { EBA-Lernende Gastronomie } \\
\text { und Detailhandel }\end{array}$ & $\begin{array}{l}\mathrm{T} 1 \\
\mathrm{~N}=319\end{array}$ & $\begin{array}{l}\mathrm{T} 2 \\
\mathrm{~N}=211\end{array}$ & & $\begin{array}{l}\mathrm{T} 3 \\
\mathrm{~N}=170\end{array}$ & & \\
\hline $\begin{array}{l}\text { EBA-Lernende Schreinerei } \\
\text { und Hauswirtschaft }\end{array}$ & & & $\begin{array}{l}\mathrm{T} 1 \\
\mathrm{~N}=206\end{array}$ & $\begin{array}{l}\mathrm{T} 2 \\
\mathrm{~N}=141\end{array}$ & & $\begin{array}{l}\mathrm{T} 3 \\
\mathrm{~N}=98\end{array}$ \\
\hline $\begin{array}{l}\text { Lehrpersonen } \\
\text { Betriebliche Berufsbildende } \\
\text { Arbeitgebende }\end{array}$ & $\begin{array}{l}\mathrm{N}=43 \\
\mathrm{~N}=47\end{array}$ & $\mathrm{~N}=30$ & $\begin{array}{l}\mathrm{N}=21 \\
\mathrm{~N}=65\end{array}$ & $\mathrm{~N}=40$ & & \\
\hline
\end{tabular}

Die Erstbefragungen der EBA-Lernenden (2007) in den Branchen Gastronomie/ Detailhandel und in der Schreinerei/Hauswirtschaft (2009) fanden kurz vor Ende ihrer Ausbildung statt und wurden schriftlich im Klassenverband durchgeführt. Ergänzend dazu wurden Einschätzungen von Lehrpersonen und betrieblichen Berufsbildenden erhoben (Hofmann \& Häfeli, 2010; Hofmann \& Kammermann, 2009; Kammermann, Amos, Hofmann, \& Hättich, 2009). Ein Jahr nach Ausbildungsabschluss konnten rund zwei Drittel der ehemaligen Lernenden $(\mathrm{N}=352)$ wieder erreicht und telefonisch interviewt werden. Gleichzeitig fanden Telefoninterviews mit arbeitgebenden Betrieben statt. Zum dritten Befragungszeitpunkt (rund drei Jahre nach Ausbildungsabschluss) nahmen 268 Befragte wieder teil (51.1\%) Zusätzliche Analysen ergaben keine systematischen Ausfälle bezogen auf die involvierten Variablen.

Der schriftliche Fragebogen der Erstbefragung (T1) und die telefonischen Befragungen (T2, T3) wurden in Anlehnung an die TREE-Befragung erstellt (Stalder, Meyer, \& Hupka-Brunner, 2011) und enthielten folgende Skalen bzw. Items (vgl. Tabelle 2): 
Tabelle 2 Quellen und Kennwerte der verwendeten Skalen und Items.

\begin{tabular}{|c|c|c|c|c|}
\hline \multicolumn{2}{|l|}{ Skalen/Items } & Quellen & $\begin{array}{l}\text { Anzahl } \\
\text { Items }\end{array}$ & $\begin{array}{l}\text { Cron- } \\
\text { bach- } \alpha\end{array}$ \\
\hline \multicolumn{2}{|c|}{$\begin{array}{l}\text { Sozioökonomischer Status } \\
\text { Familie (ISEI) }\end{array}$} & $\begin{array}{l}\text { (Kammermann, Hofmann, } \\
\text { \& Hättich, 2009) }\end{array}$ & 1 & - \\
\hline \multicolumn{2}{|c|}{ Migrationshintergrund } & $\begin{array}{l}\text { (Kammermann, Hofmann, } \\
\text { et al., 2009) }\end{array}$ & 1 & - \\
\hline \multicolumn{2}{|c|}{ Schulische Herkunft } & $\begin{array}{l}\text { (Kammermann, Hofmann, } \\
\text { et al., 2009) }\end{array}$ & 1 & - \\
\hline $\begin{array}{l}\text { Unterstüt- } \\
\text { zung }\end{array}$ & $\begin{array}{l}\text { Berufsfachschule } \\
\text { Lehrbetrieb }\end{array}$ & (Frese, 1999; Zapf, 1983) & $\begin{array}{l}2 \\
2\end{array}$ & $\begin{array}{l}0.76 \\
0.83\end{array}$ \\
\hline Belastung & $\begin{array}{l}\text { Berufsfachschule } \\
\text { Lehrbetrieb }\end{array}$ & $\begin{array}{l}\text { (Prümper, Hartmannsgruber, } \\
\text { \& Frese, 1995; Semmer, Zapf, } \\
\text { \& Dunckel, 1999) }\end{array}$ & $\begin{array}{l}5 \\
5\end{array}$ & $\begin{array}{l}0.80 \\
0.72\end{array}$ \\
\hline Klima & $\begin{array}{l}\text { Berufsfachschule } \\
\text { Lehrbetrieb }\end{array}$ & (Neuenschwander, 1998) & $\begin{array}{l}3 \\
3\end{array}$ & $\begin{array}{l}0.78 \\
0.84\end{array}$ \\
\hline Vielseitigkeit & $\begin{array}{l}\text { Berufsfachschule } \\
\text { Lehrbetrieb }\end{array}$ & $\begin{array}{l}\text { (Prümper et al., 1995; Semmer } \\
\text { et al., 1999) }\end{array}$ & $\begin{array}{l}3 \\
3\end{array}$ & $\begin{array}{l}0.62 \\
0.75\end{array}$ \\
\hline $\begin{array}{l}\text { Leistungen } \\
\text { (Selbstein- } \\
\text { schätzung) }\end{array}$ & $\begin{array}{l}\text { Berufsfachschule } \\
\text { Lehrbetrieb }\end{array}$ & $\begin{array}{l}\text { (Kammermann, Hofmann, } \\
\text { et al., 2009) }\end{array}$ & $\begin{array}{l}2 \\
1\end{array}$ & $\begin{array}{l}0.78 \\
-\end{array}$ \\
\hline \multicolumn{2}{|l|}{ Selbstwert } & (Rosenberg, 1979) & 4 & 0.72 \\
\hline \multicolumn{2}{|l|}{ Depressivität } & (Rosenberg, 1979) & 4 & 0.76 \\
\hline \multicolumn{2}{|c|}{ Berufliche Verbundenheit } & (Neuenschwander, 1998) & 4 & 0.86 \\
\hline \multicolumn{2}{|c|}{ Arbeitswerte intrinsisch } & (Watermann, 2000) & 5 & 0.74 \\
\hline \multicolumn{2}{|c|}{ Arbeitswerte extrinsisch } & (Watermann, 2000) & 4 & 0.62 \\
\hline \multicolumn{2}{|c|}{ (Arbeits-)zufriedenheit (T2, T3) } & (Baillod, 1992) & 1 & - \\
\hline \multicolumn{2}{|c|}{ Arbeitssituation (T2, T3) } & $\begin{array}{l}\text { (Kammermann, Hofmann, } \\
\text { et al., 2009) }\end{array}$ & 1 & - \\
\hline
\end{tabular}

\subsection{Beschreibung der Stichproben}

Zum ersten Befragungszeitpunkt betrug das Durchschnittsalter der EBA-Lernenden 19.7 Jahre ( $\mathrm{SD}=2.1$ Jahre). Rund zwei Drittel der Befragten sind weiblich (62.5\%), allerdings mit grossen Branchenunterschieden (Hauswirtschaft 96.2\%, Detailhandel 78.4\%, Gastronomie 53.7\% Schreinerpraktiker/-innen 9\%). 
Die folgenden Ergebnisse basieren auf den Angaben von 525 EBA-Lernenden zum ersten Befragungszeitpunkt (ohne Einbezug der Anlehrlinge), d.h. 185 angehenden Detailhandelsassistenten/-innen (35.2\%), 134 Küchen- bzw. Restaurations- bzw. Hotelangestellten (25.5\%), 106 Hauswirtschaftspraktiker/-innen (20.2\%) und 100 Schreinerpraktiker/-innen (19.0\%). Die Mehrheit der Befragten kommt aus der Deutschschweiz (83,4\%), 11\% aus der französischsprachigen Schweiz und 5.5\% aus dem Kanton Tessin.

\section{$4 \quad$ Ergebnisse}

\subsection{Schulische Herkunft und Ausbildungssituation}

In der Hauswirtschaft ist der Anteil der Lernenden aus Sonderklassen/-schulen mit 51.0\% am höchsten, gefolgt von der Schreinerei mit 21.4\%, der Gastronomie mit 20.2\% und dem Detailhandel mit nur 8.4\%. Für die Branchen Detailhandel und Gastronomie zeigt ein Vergleich mit einer früheren Befragung der Anlehrlinge (Hofmann \& Kammermann, 2009), dass der Anteil Lernender aus Sonderklassen/-schulen deutlich abgenommen hat (im Detailhandel von 34.7\% auf 8.4\%, in der Gastronomie von 46.8\% auf 20.2\%). Mehr als zwei Drittel der EBA-Lernenden absolvierten vor Ausbildungsbeginn eine oder mehrere Zwischenlösungen. Unterschiede nach schulischer Herkunft lassen sich bei dieser Frage nicht feststellen.

Die meisten EBA-Lernenden (85\%) haben ihre Ausbildung in einem Lehrbetrieb im ersten Arbeitsmarkt absolviert, 15\% in einem geschützten Rahmen. Vor allem in der Hauswirtschaft ist der Anteil der Lernenden im geschützten Umfeld mit 37\% relativ hoch (gefolgt von der Gastronomie mit 21.1\%, der Schreinerei mit 14\% und dem Detailhandel mit 1.1\%). Die schulische Herkunft beeinflusst erwartungsgemäss die Wahl des Ausbildungsumfelds: 89\% der ehemaligen Regelklässler/-innen absolvierten ihre Ausbildung im ersten Arbeitsmarkt gegenüber 66\% in der Gruppe der Lernenden aus Sonderklassen bzw. -schulen. Abbildung 2 zeigt, wie die EBA-Lernenden (differenziert nach schulischer Herkunft) verschiedene Ausbildungsaspekte im Rückblick bewerten. 


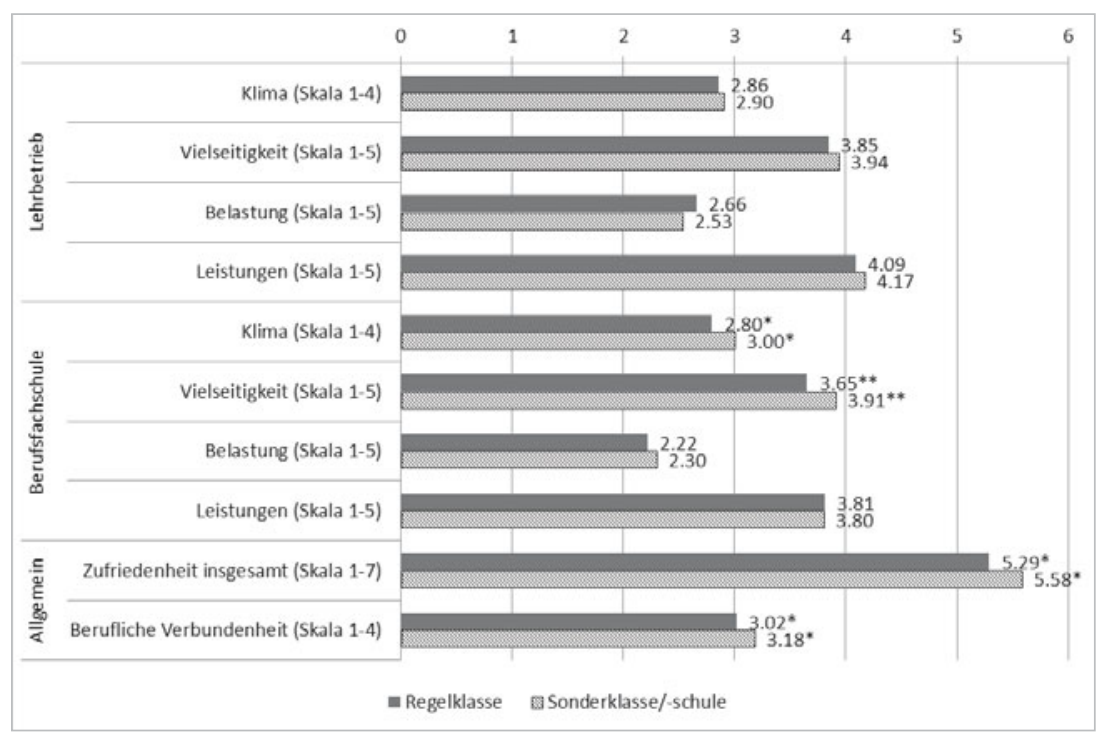

Abbildung 2 Bewertung der Situation im Lehrbetrieb und in der Berufsfachschule nach schulischer Herkunft $(\mathrm{N}=525),{ }^{*}=$ auf dem $5 \%$-Niveau sign. Unterschied, ${ }^{* *}$ auf dem 1-\% Niveau sign. Unterschied.

Sowohl Lernende aus Sonderschulen/-klassen wie auch Lernende aus Regelklassen beurteilen die Ausbildung positiv. Lernende, die ehemals Sonderklassen/schulen besuchten, empfanden das Klima in der Berufsfachschule sogar als noch etwas angenehmer (T-Test, $\mathrm{t}=-2.27, \mathrm{df}=505, \mathrm{p}<0.05$ ), den Unterricht als vielseitiger (T-Test, $\mathrm{t}=-3.70, \mathrm{df}=506, \mathrm{p}<0.001$ ), fühlten sich stärker mit dem erlernten Beruf verbunden (T-Test, $\mathrm{t}=-2.11, \mathrm{df}=499, \mathrm{p}<0.05)$ und waren auch insgesamt mit der Ausbildung zufriedener (T-Test, $\mathrm{t}=-2.33, \mathrm{df}=508, \mathrm{p}<0.05)$. Bezüglich Branchen ist festzustellen, dass Lernende aus der Schreinerei im Vergleich mit den anderen Branchen ihre Arbeit im Lehrbetrieb weniger vielseitig finden, ihre Leistungen kritischer einschätzen und das Klima in der Schule ungünstiger beurteilen. Lernende aus der Gastronomie fühlen sich ihrem Beruf verbundener und erleben die Schule als vielseitiger. Lernende aus der Hauswirtschaft beurteilen v.a. die Situation in der Berufsfachschule positiver (vielseitiger, bessere Leistungseinschätzung, weniger belastet). Zusätzlich zeigte sich, dass v.a. in der Gastronomie die Lernenden aus Sonderklassen/-schulen viele Aspekte im Vergleich mit den Regelklässler/-innen unterschiedlich beurteilen: Sie sind allgemein zufriedener, fühlen sich ihrem Beruf verbundener, empfinden den Unterricht und die Arbeit 
im Betrieb als vielseitiger, fühlen sich allerdings an beiden Lernorten stärker belastet. In den anderen Branchen unterscheiden sich die Beurteilungen jeweils nur in einzelnen Punkten.

Vor dem Hintergrund der weitgehend positiven Beurteilung der Ausbildungssituation stellt sich die Frage, ob sie ein Indikator dafür ist, dass die zusätzliche Unterstützung durch die fachkundige individuelle Begleitung (FiB) greift. Allerdings hatte nur rund ein Drittel zu den damaligen Zeitpunkten $(2007,2009)$ von diesem Angebot Kenntnis (unabhängig von der Branche und der schulischen Herkunft). Von diesen Personen haben etwas mehr als die Hälfte (54\%) eine FiB in Anspruch genommen. Bei den Sonderklässler/-innen sind es mit 75\% anteilsmässig signifikant mehr im Vergleich mit $48 \%$ bei den Regelklässler/-innen (Pearson Chi-Quadrat, $\chi^{2}=8.27, \mathrm{df}=1, \mathrm{p}>0.01$ ). Die Stellungnahmen der Lehrpersonen waren bezüglich Unterstützungsmöglichkeiten kritisch: Je nach Branche waren $40 \%$ (Schreinerei) bis zu 61\% (Detailhandel) der Meinung, dass Leistungsschwächere trotz individueller Begleitung Mühe haben, den schulischen Anforderungen gerecht zu werden. Betriebliche Berufsbildende waren in diesem Punkt jedoch optimistischer.

\subsection{Berufliche Situation nach Ausbildungsabschluss}

Ein Jahr nach Ausbildungsabschluss (T2) sind mehr als vier Fünftel der ehemaligen EBA-Lernenden in irgendeiner Form beruflich integriert, zwei Jahre später (T3) sind es bereits mehr als 85\%. Die berufliche Situation nach Ausbildungsabschluss unterscheidet sich deutlich zwischen den Branchen. Die folgende Abbildung 3 zeigt die berufliche Situation differenziert nach schulischer Herkunft: 


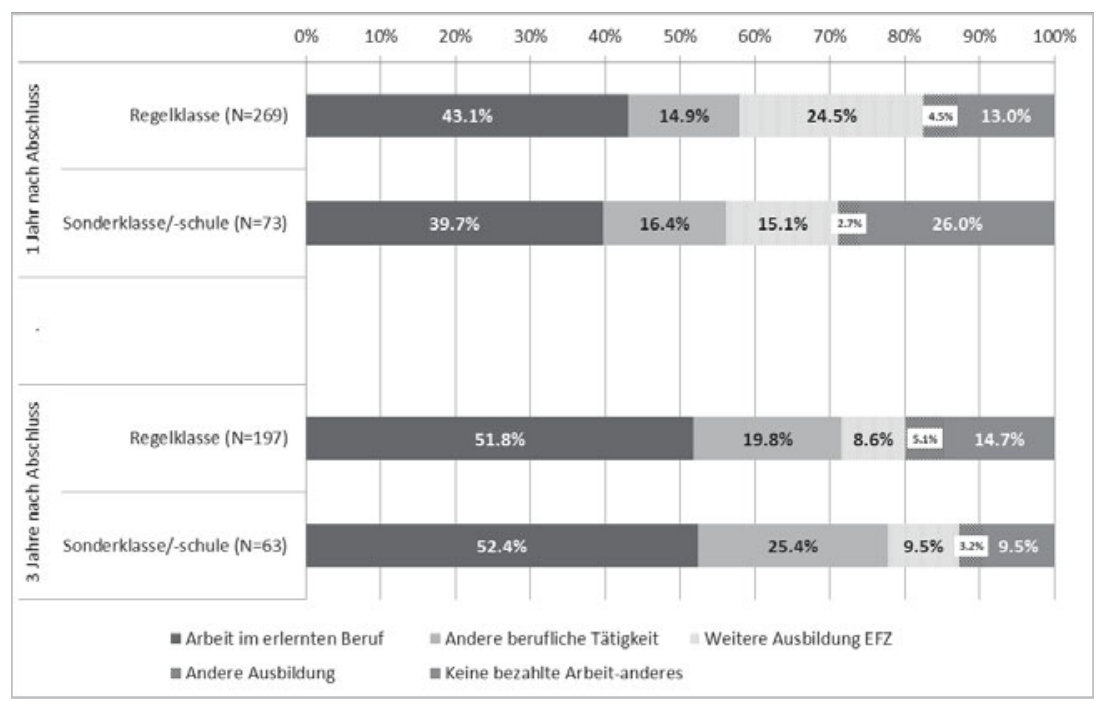

Abbildung 3 Berufliche Situation nach schulischer Herkunft ein Jahr $(\mathrm{N}=352)$ und rund drei Jahre (N=268) nach Ausbildungsabschluss.

Wird nun nach schulischer Herkunft unterschieden, präsentiert sich die Situation der jungen Erwachsenen aus Sonderklassen oder -schulen ein Jahr nach Ausbildungsabschluss etwas ungünstiger als bei der Gruppe aus Regelklassen (vgl. Abbildung 3): Doppelt so viele sind ohne Erwerbsarbeit (26\%) und mit 15\% nehmen deutlich weniger eine EFZ-Ausbildung in Angriff (im Vergleich zu 25\% bei den Regelklässer/-innen) (Pearson Chi-Quadrat, $\chi 2=8.90 \mathrm{df}=2$, $\mathrm{p}<0.05$ ). Bei der Gruppe aus Regelklassen haben mehr als drei Viertel eine Festanstellung, bei jener aus Sonderklassen oder -schulen sind es rund zwei Drittel (Pearson-Chi-Quadrat, n.s.). Ehemalige Regelklässler/-innen verdienen mit durchschnittlich 3698 SFr. (brutto, bei einer Vollzeitanstellung, Median=3617 SFr. ${ }^{1}$ ) mehr als Sonderklässler/-innen und -schüler/-innen mit rund $3066 \mathrm{SFr}$. (Median $=3478 \mathrm{SFr}$.) ${ }^{2}$ (T-Test, $\mathrm{t}=3.76, \mathrm{df}=186, \mathrm{p}<0.001)$. Das Arbeitspensum unterscheidet sich dagegen nicht: In beiden Gruppen arbeiten rund drei Viertel Vollzeit.

1 Der Median eignet sich hier als zusätzliches Vergleichsmass, weil er weniger sensibel auf Extremwerte reagiert. Deshalb liegt der Medianwert bei den ehemaligen Sonderklässler/-innen, die häufiger im zweiten Arbeitsmarkt mit tiefem Einkommen arbeiten, deutlich höher als der Durchschnittswert.

2 Es wurden Löhne im ersten und im zweiten Arbeitsmarkt einbezogen. 
Knapp drei Jahre nach Ausbildungsabschluss hat sich die berufliche Situation vor allem für die jungen Erwachsenen aus Sonderklassen und -schulen stabilisiert, d.h. der Anteil von Personen ohne Erwerbstätigkeit ist in dieser Gruppe deutlich zurückgegangen und mehr als $90 \%$ sind inzwischen festangestellt (vergleichbar mit den ehemaligen Regelklässler/-innen). Es fällt jedoch auf, dass bereits $25.4 \%$ der Befragten aus Sonderklassen oder -schulen nicht mehr im erlernten Beruf arbeiten (aus Regelklassen: 19\%). Nach wie vor verdienen sie auch weniger als ihre Ausbildungskollegen/-innen aus Regelklassen (durchschnittlich 3476 SFr. brutto bei Vollzeit/ Median=3619 SFr. gegenüber 3915 SFr. brutto, Median=3917 SFr., T-Test, $\mathrm{t}=2.72, \mathrm{df}=179, \mathrm{p}>0.01$ ). Diese Ergebnisse bestätigen sich auch in Analysen, die nur mit denjenigen Lernenden durchführt wurden, die zu beiden Erhebungszeitpunkten (T2, T3) an der Befragung teilgenommen haben (gleiche Stichprobengrössen).

Im Weiteren stellt sich die Frage, wie es um die subjektive Einschätzung der beruflichen Situation steht: Insgesamt ist die (berufliche) Zufriedenheit hoch (Mittelwert T2=5.2 bzw. T3=5.1 auf einer Skala von 1-7). Ein Jahr nach Ausbildungsabschluss sind ehemalige Regelklässler/-innen tendenziell etwas zufriedener als Befragte aus Sonderklassen oder -schulen (T-Test, $\mathrm{t}=1.65, \mathrm{df}=330, \mathrm{p}=0.10)^{3}$. Rund drei Jahre nach Ausbildungsabschluss hat die Zufriedenheit in dieser Gruppe zugenommen und ist zu diesem Zeitpunkt tendenziell höher als bei derjenigen aus Regelklassen ( $\mathrm{T}-T e s t, \mathrm{t}=-1.91, \mathrm{df}=256, \mathrm{p}=0.058$ ). Auch diese Ergebnisse werden bestätigt, wenn mit der reduzierten Stichprobe (Angaben über alle drei Zeitpunkte) gerechnet wird.

Bezüglich Branchen ist festzustellen, dass im Detailhandel der Anteil derjenigen, die noch im erlernten Beruf arbeiten, zu beiden Erhebungszeitpunkten am höchsten (T2=54\%, T3=71\%) ist. Ebenfalls am höchsten ist in dieser Branche der Anteil derjenigen, die eine EFZ-Ausbildung anschliessen (30\%). Der Anteil Erwerbsloser ist ein Jahr nach Abschluss in der Hauswirtschaft mit 19\% und drei Jahre danach in der Gastronomie (mit 24\%) am höchsten. Weitere Analysen zeigen, dass die höhere Erwerbslosigkeit von Lernenden aus Sonderklassen/-schulen zu T2 v.a. auf die Gastronomiebranche zurückzuführen ist. In der Hauswirtschaft sind Lernende aus Regelklassen zu T3 besonders häufig in einer anderen Ausbildung oder auch erwerbslos. Die tiefere Zufriedenheit der ehemaligen Sonderklässler/-innen zu T2 findet sich tendenziell in allen Branchen. Eine statistisch

3 Dieser Unterschied besteht allerdings nicht in der Teilgruppe derjenigen, die zu diesem Zeitpunkt (T2) arbeiten oder in Ausbildung sind, hat also vermutlich mit der höheren Erwerbslosigkeit von ehemaligen Sonderklässler/-innen zu tun. 
signifikant höhere Zufriedenheit ist zu T3 in den Branchen Gastronomie und Schreinerei zu finden.

\subsection{Determinanten des objektiven und des subjektiven Laufbahnerfolgs}

Mittels logistischer Regression wurde errechnet, wie sich verschiedenen Faktoren (vgl. Kap. 2.2.) auf die Wahrscheinlichkeit auswirken, keiner Erwerbstätigkeit nachzugehen bzw. eine weiterführende Ausbildung zu absolvieren (vgl. Tabellen 3 und 4). Bei den folgenden Analysen (Tab. 3 und Tab. 4) wurden wiederum unterschiedliche Stichproben für T2 und T3 einbezogen, jedoch bestätigen sich die Ergebnisse im Wesentlichen, wenn man nur diejenigen einbezieht, die an beiden Messzeitpunkten teilnahmen.

Auch wenn andere Faktoren kontrolliert werden, hat die schulische Herkunft einen Einfluss darauf, ob jemand ein Jahr nach Ausbildungsabschluss einer Erwerbstätigkeit nachgeht oder nicht (Tabelle 3): Bei jungen Berufsleuten aus Sonderklassen oder -schulen ist die Wahrscheinlichkeit erwerbslos zu sein rund 2.5 Mal so hoch. Zwei weitere Variablen haben diesbezüglich tendenziell einen Einfluss: Je vielseitiger die Aufgaben im Lehrbetrieb beurteilt wurden, desto geringer ist die Wahrscheinlichkeit ein Jahr nach Abschluss ohne Erwerbsarbeit zu sein und je depressiver sich eine Person beschreibt, desto höher ist diese Wahrscheinlichkeit. Bei der Befragung drei Jahr nach Abschluss spielen diese drei Variablen keine Rolle mehr. Vielmehr zeigt sich ein bedeutsamer Einfluss der extrinsischen Arbeitswerte: Je wichtiger diese eingeschätzt wurden, desto geringer die Wahrscheinlichkeit, keiner Erwerbstätigkeit nachzugehen. 
Tabelle 3 Einflussfaktoren auf die Wahrscheinlichkeit, nach Ausbildungsabschluss keiner bezahlten Erwerbsarbeit nachzugehen (logistische Regression).

\begin{tabular}{|c|c|c|c|c|}
\hline \multirow[t]{2}{*}{ T1-Prädiktoren } & \multicolumn{2}{|c|}{$\begin{array}{l}\text { T2- } 1 \text { Jahr nach } \\
\text { Abschluss }(\mathrm{N}=280)\end{array}$} & \multicolumn{2}{|c|}{$\begin{array}{l}\text { T3- } 3 \text { Jahre nach } \\
\text { Abschluss }(\mathrm{N}=211)\end{array}$} \\
\hline & B & $\operatorname{Exp}(B)$ & B & $\operatorname{Exp}(B)$ \\
\hline Sozioök. Status der Familie (ISEI) & 0.01 & 1.01 & 0.01 & 1.01 \\
\hline $\begin{array}{l}\text { Migrationshintergrund } \\
\text { (1=keiner - } 5=\text { weniger als } 7 \text { Jahre } \\
\text { /Schweiz) }\end{array}$ & 0.16 & 1.18 & 0.24 & 1.28 \\
\hline $\begin{array}{l}\text { Schulische Herkunft } \\
\text { (1=Regelklasse, } 2=\text { Sonderklasse/- } \\
\text { schule) }\end{array}$ & $0.91^{*}$ & $2.49^{\star}$ & -0.59 & 0.56 \\
\hline $\begin{array}{l}\text { Unterstützung Berufsbildner } \\
\text { Betrieb }\end{array}$ & -0.02 & 0.98 & -0.12 & 0.89 \\
\hline Unterstützung Lehrperson & -0.09 & 0.92 & -0.06 & 0.94 \\
\hline $\begin{array}{l}\text { Vielseitigkeit der Arbeit im Be- } \\
\text { trieb }\end{array}$ & $-0.51^{t}$ & $0.60^{t}$ & 0.16 & 1.17 \\
\hline Belastung in der Schule & -0.04 & 0.96 & 0.28 & 1.32 \\
\hline Selbstwert & 0.44 & 1.55 & 0.47 & 1.60 \\
\hline Depressivität & $0.42^{\mathrm{t}}$ & $1.52^{\mathrm{t}}$ & 0.38 & 1.46 \\
\hline Leistungen Schule & -0.06 & 0.94 & 0.20 & 1.22 \\
\hline Leistungen Betrieb & -0.10 & 0.91 & 0.06 & 1.06 \\
\hline Arbeitswerte intrinsisch & 0.33 & 1.39 & 0.93 & 2.53 \\
\hline \multirow[t]{2}{*}{ Arbeitswerte extrinsisch } & -.032 & 0.73 & $-1.18^{\star}$ & $0.31^{*}$ \\
\hline & \multicolumn{2}{|c|}{$\begin{array}{l}\mathrm{R}^{2}=.10(\text { Nagelkerke }) \\
\mathrm{Chi}^{2}=15.26(\mathrm{df}=13)\end{array}$} & \multicolumn{2}{|c|}{$\begin{array}{l}\mathrm{R}^{2}=.13 \text { (Nagelkerke) } \\
\mathrm{Chi}^{2}=14.11(\mathrm{df}=13)\end{array}$} \\
\hline
\end{tabular}

Anmerkungen ${ }^{*} \mathrm{p}<0.05,{ }^{\mathrm{t}} \mathrm{p}<0.10$ 
Tabelle 4 Einflussfaktoren auf die Wahrscheinlichkeit, nach Ausbildungsabschluss eine Weiterbildung (EFZ) anzuschliessen (logistische Regression).

\begin{tabular}{|c|c|c|c|c|}
\hline \multirow[t]{2}{*}{ T1-Prädiktoren } & \multicolumn{2}{|c|}{$\begin{array}{l}\text { T2- } 1 \text { Jahr nach } \\
\text { Abschluss }(\mathrm{N}=280)\end{array}$} & \multicolumn{2}{|c|}{$\begin{array}{l}\text { T3- } 3 \text { Jahre nach } \\
\text { Abschluss }(\mathrm{N}=211)\end{array}$} \\
\hline & B & $\operatorname{Exp}(B)$ & B & $\operatorname{Exp}(B)$ \\
\hline Sozioök. Status der Familie (ISEI) & -0.01 & 1.00 & -0.01 & 0.99 \\
\hline $\begin{array}{l}\text { Migrationshintergrund } \\
\text { (1=keiner - 5=weniger als } 7 \text { Jahre / } \\
\text { Schweiz) }\end{array}$ & -0.17 & 0.84 & -0.19 & 0.83 \\
\hline $\begin{array}{l}\text { Schulische Herkunft } \\
\text { (1=Regelklasse, } 2=\text { Sonderklasse/- } \\
\text { schule) }\end{array}$ & -0.55 & 0.58 & $-0.80^{t}$ & $0.45^{\mathrm{t}}$ \\
\hline Unterstützung Berufsbildner Betrieb & 0.08 & 1.08 & 0.31 & 1.37 \\
\hline Unterstützung Lehrperson & 0.01 & 1.01 & 0.18 & 1.12 \\
\hline Vielseitigkeit der Arbeit im Betrieb & 0.12 & 1.13 & -0.24 & 0.79 \\
\hline Belastung in der Schule & $-0.44^{\mathrm{t}}$ & $0.64^{\mathrm{t}}$ & -0.30 & 0.74 \\
\hline Selbstwert & 0.01 & 1.01 & -0.14 & 0.87 \\
\hline Depressivität & 0.09 & 0.91 & -0.08 & 0.92 \\
\hline Leistungen Schule & $0.51^{*}$ & $1.66^{*}$ & $0.68^{\star}$ & $1.97^{\star}$ \\
\hline Leistungen Betrieb & 0.34 & 1.40 & -0.15 & 0.86 \\
\hline Arbeitswerte intrinsisch & -0.03 & 0.97 & -0.01 & 0.99 \\
\hline \multirow[t]{2}{*}{ Arbeitswerte extrinsisch } & 0.30 & 1.35 & 0.65 & 1.91 \\
\hline & \multicolumn{2}{|c|}{$\begin{array}{l}\mathrm{R}^{2}=.14 \text { (Nagelkerke) } \\
\text { Chi }^{2}=28.70(\mathrm{df}=13)\end{array}$} & \multicolumn{2}{|c|}{$\begin{array}{l}\mathrm{R}^{2}=.16 \text { (Nagelkerke) } \\
\mathrm{Chi}^{2}=25.43(\mathrm{df}=13)\end{array}$} \\
\hline
\end{tabular}

Anmerkungen ${ }^{*} \mathrm{p}<0.05,{ }^{\mathrm{t}} \mathrm{p}<0.10$

Betrachtet man als weiteres objektives Kriterium die Wahrscheinlichkeit, eine berufliche Weiterbildung in Angriff zu nehmen (vgl. Tab. 4), so spielen hier v.a. die Leistungen und die Belastung in der Berufsfachschule eine Rolle. Je höher die Schulleistungen eingeschätzt werden, desto höher die Wahrscheinlichkeit, dass jemand in einer Weiterbildung ist. Je höher die schulische Belastung, desto geringer ist diese Wahrscheinlichkeit. Für T3 (drei Jahre nach Abschluss) zeigt sich zudem, dass junge Berufsleute aus Sonderklassen/-schulen weniger in einer beruflichen Weiterbildung sind bzw. eine solche bereits abgeschlossen haben. 
Wird schliesslich die berufliche Zufriedenheit nach Ausbildungsabschluss betrachtet, so zeigt sich: Die schulische Herkunft übt zu beiden Nachbefragungszeitpunkten einen Einfluss aus, allerdings in gegenläufiger Richtung (vgl. Tabelle 5):

Tabelle 5 Einflussfaktoren auf die (berufliche) Zufriedenheit nach Ausbildungsabschluss (lineare Regression).

\begin{tabular}{|c|c|c|}
\hline T1-Prädiktoren & $\begin{array}{l}\text { T2- } 1 \text { Jahr nach } \\
\text { Abschluss }(\mathrm{N}=269)\end{array}$ & $\begin{array}{l}\text { T3- } 3 \text { Jahre nach } \\
\text { Abschluss }(\mathrm{N}=208)\end{array}$ \\
\hline & $\beta$ & $\beta$ \\
\hline Sozioök. Status der Familie (ISEI) & -0.07 & -0.03 \\
\hline $\begin{array}{l}\text { Migrationshintergrund } \\
\text { (1=keiner - 5=weniger als } 7 \text { Jahre / } \\
\text { Schweiz) }\end{array}$ & -0.07 & -0.02 \\
\hline $\begin{array}{l}\text { Schulische Herkunft } \\
\text { (1=Regelklasse, } 2=\text { Sonderklasse/- } \\
\text { schule) }\end{array}$ & $-0.12^{\star}$ & $0.14^{*}$ \\
\hline Unterstützung Berufsbildner Betrieb & 0.07 & -0.04 \\
\hline Unterstützung Lehrperson & 0.05 & 0.11 \\
\hline Vielseitigkeit der Arbeit im Betrieb & 0.04 & 0.10 \\
\hline Belastung in der Schule & $-0.17^{\star}$ & -0.07 \\
\hline Selbstwert & 0.01 & -0.01 \\
\hline Depressivität & -0.11 & -0.11 \\
\hline Leistungen Schule & -0.05 & -0.05 \\
\hline Leistungen Betrieb & 0.11 & -0.03 \\
\hline Arbeitswerte intrinsisch & -0.04 & $-0.17^{\star}$ \\
\hline \multirow[t]{2}{*}{ Arbeitswerte extrinsisch } & 0.04 & 0.02 \\
\hline & $\begin{array}{l}\mathrm{R}^{2}=.11, \mathrm{~F}=2.61, \mathrm{df}=12 \\
\mathrm{p}<0.01\end{array}$ & $\begin{array}{l}\mathrm{R}^{2}=.06, \mathrm{~F}=1.03, \mathrm{df}=12 \\
\mathrm{p}<0.50\end{array}$ \\
\hline
\end{tabular}

Anmerkungen ${ }^{\star} \mathrm{p}<0.05,{ }^{\mathrm{t}} \mathrm{p}<0.10$

Ein Jahr nach Ausbildungsabschluss sind junge Berufsleute aus Sonderklassen/schulen mit ihrer Situation unzufriedener. Rund drei Jahre nach Ausbildungsabschluss sind sie hingegen signifikant zufriedener mit ihrer beruflichen Situation. 
Ein Jahr nach Abschluss wirkt sich weiter die erlebte schulische Belastung negativ auf die Arbeitszufriedenheit aus und drei Jahre danach sind junge Berufsleute unzufriedener, die am Ende der Ausbildung höhere intrinsische Werthaltungen zur Arbeit aufwiesen. Die aufgeklärte Varianz ist besonders zu diesem Zeitpunkt ungenügend, d.h. dass nun offensichtlich viele andere wesentliche Faktoren die Arbeitszufriedenheit beeinflussen.

\section{$5 \quad$ Zusammenfassung und Schlussfolgerungen}

Die Einführung der zweijährigen Grundbildungen mit eidgenössischem Berufsattest startete mit dem Anspruch, die Lernenden mit besseren Chancen in den Arbeitsmarkt zu entlassen. Dies führte, aus Sicht der beteiligten Berufsbildenden, in vielen Branchen zu erhöhten Ausbildungsanforderungen. Gleichzeitig bleibt das bildungspolitische Ziel prioritär, bis ins Jahr 2015 95\% der Jugendlichen einen Berufsabschluss zu ermöglichen. Nach rund zehn Jahren Erfahrung mit dem neuen Ausbildungsgefäss stellt sich die Frage, inwieweit die Gratwanderung gelungen ist, einerseits den Bedürfnissen der Schwächeren in der Berufsbildung gerecht zu werden und andererseits die Stärkeren optimal auf den Arbeitsmarkt vorzubereiten. Dies zeigt sich unter anderem daran, wie gut es gelingt, Jugendliche aus Sonderklassen und -schulen im Ausbildungssystem so zu integrieren, dass sie zufrieden und leistungsfähig sind und mit vergleichbaren Chancen in den Arbeitsmarkt starten.

Insgesamt zeichnen die Ergebnisse ein günstiges Bild der EBA-Grundbildungen aus Sicht der befragten Lernenden: Sie sind überwiegend zufrieden mit ihrer Ausbildungssituation und fühlen sich nicht übermässig belastet. Dies gilt insbesondere auch für ehemalige Lernende aus Sonderklassen und -schulen. In einigen Punkten beurteilen sie ihre Ausbildungssituation (v.a. in der Berufsfachschule) sogar positiver. Entscheidend ist aber v.a., dass sie sich weder in der Berufsfachschule noch im Betrieb stärker belastet fühlen als ihre Kollegen/-innen aus der Regelklasse und ihre Leistungen in beiden Lernumfeldern vergleichbar positiv einschätzen. Die Passung zwischen Ausbildungsangebot einerseits und Möglichkeiten und Bedürfnissen der befragten Gruppe andererseits scheint somit gelungen zu sein.

In zwei Punkten ist diese Einschätzung jedoch zu relativieren: Zum einen zeigt der Vergleich mit der ehemaligen Anlehre (Gastronomie und Detailhandel), dass sich die Zusammensetzung der Lernenden bezüglich schulischer Herkunft stark verändert hat. Es ist deshalb anzunehmen, dass es für Jugendliche aus Sonderklassen/-schulen trotz des grösseren Ausbildungsangebots schwieriger geworden 
ist, einen Ausbildungsplatz zu finden. Befragungen der betrieblichen Berufsbildenden zu den EBA-Ausbildungen (Hofmann \& Kammermann, 2008) weisen jedenfalls darauf hin, dass rund zwei Drittel der Betriebe ihre Selektionspraxis nach der Einführung der EBA-Ausbildung den höheren schulischen Anforderungen angepasst haben. Dies erklärt auch den hohen Anteil von Lernenden, die gemäss unserer Analysen eine oder mehrere Zwischenlösungen absolviert haben (im Vergleich dazu schaffen je nach Kanton 83-98\% den direkten Übertritt in eine EFZ-Berufsausbildung, vgl. Keller \& Moser (2013)). Unterschiede nach schulischer Herkunft lassen sich bei dieser Frage allerdings nicht feststellen. Jedoch absolvieren deutlich mehr Lernende aus Sonderklassen/-schulen ihre Ausbildungen im geschützten Rahmen, was wiederum ihre Arbeitsmarktchancen nach Abschluss höchst wahrscheinlich beeinträchtigt. Zum zweiten ist es wichtig darauf hinzuweisen, dass mit der Befragung am Ende der Ausbildungszeit diejenigen Lernenden ausgeschlossen wurden, die nicht bis zum Abschluss durchgehalten haben. Dies erklärt auch die deutlich kritischeren Rückmeldungen der Lehrpersonen zur Situation und zu den Unterstützungsmöglichkeiten, die diese „gescheiterten" Lernenden in ihrer Stellungnahme miteinbezogen.

Ein gewichtiges Argument für die Einführung der EBA-Ausbildung war die Verbesserung der Arbeitsmarktfähigkeit und Durchlässigkeit zu weiterführenden Aus- und Weiterbildungen. Unsere Ergebnisse zeigen, dass ein Jahr nach Ausbildungsschluss mehr als vier Fünftel beruflich integriert sind, knapp zwei Jahre später sind es bereits mehr als $85 \%$. Verglichen mit der Integrationsquote nach der Anlehre hat sich die Situation zumindest in den beiden untersuchten Branchen Detailhandel und Gastronomie verbessert. Ausserdem nehmen mehr Jugendliche eine EFZ-Ausbildung in Angriff (vgl. Kammermann, Amos, et al., 2009). Die Frage war nun, ob der standardisierte Abschluss auch dazu führt, dass die schulische Herkunft beim Eintritt in den Arbeitsmarkt an Bedeutung verliert. Diesbezüglich ist festzustellen, dass die ehemaligen Sonderklässler/-innen in der Einstiegsphase zwar ein erhöhtes Risiko haben, erwerbslos zu sein (auch unter Kontrolle anderer Faktoren). Drei Jahr später hat sich die Situation der beiden Gruppen jedoch weitgehend angeglichen. Einzig die Lohnunterschiede bleiben bestehen, die vermutlich darauf zurückzuführen sind, dass einige ehemalige Sonderschüler/-innen im geschützten Rahmen arbeiten und einen der Leistungsfähigkeit entsprechend reduzierten Lohn erhalten. Mit durchschnittlich 9.5\% (Sonderklassen/-schulen) bzw. 14.7\% sind in der untersuchten Gruppe dennoch mehr junge Berufsleute erwerbslos im Vergleich mit einer altersentsprechenden Gruppe in der Gesamtbevölkerung (vgl. z.B. Bundesamt für Statistik 2012: 3.2\%). In Bezug auf die Weiterbildung und Durchlässigkeit zum EFZ zeigen sich in den deskriptiven Analysen Unterschiede nach schulischer Herkunft. Allerdings ver- 
liert dieser Faktor etwas an Vorhersagekraft, wenn die Situation in der Berufsfachschule miteinbezogen wird: Die Belastungen und v.a. die selbsteingeschätzten Leistungen in diesem Umfeld erweisen sich hier als relevanter bzw. vermitteln vermutlich die Effekte der schulischen Herkunft.

Neben diesen objektiven Indikatoren interessierte auch, wie die jungen Berufsleute ihre aktuelle Arbeitssituation nach Abschluss subjektiv erleben. Hier zeigt sich, dass die (berufliche) Zufriedenheit der Lernenden aus Sonderklassen oder -schulen ein Jahr nach Abschluss tiefer, jedoch drei Jahre nach Abschluss höher ist. Vermutlich fällt es gerade Jugendlichen mit kognitiven oder anderen Einschränkungen schwerer, sich auf ein neues Arbeitsumfeld einzustellen. Sie sind deshalb in der Übergangsphase stärker belastet und brauchen etwas länger, um nach der gut auf sie zugeschnittenen Ausbildungssituation die Passung wieder herzustellen. Dass sie hingegen zum späteren Erhebungszeitpunkt zufriedener sind, könnte ein Ausdruck davon sein, dass sich diese jungen Berufsleute eher gewohnt sind, ihre Ansprüche an die Gegebenheiten anzupassen (Stichwort „Aspirationsabkühlung"). Ausserdem könnte die am Ende der Ausbildung höhere „berufliche Verbundenheit“ in dieser Gruppe über eine gewisse „Durststrecke“ hinweghelfen. In diesen Erklärungszusammenhang passt auch, dass sich die intrinsische Motivation am Ende der Ausbildung eher negativ auf die spätere berufliche Zufriedenheit auswirkt (ähnlich wie bei Rüfenacht \& Neuenschwander, 2014). Insgesamt heben sich diese Befunde positiv von anderen Forschungsergebnissen ab (z.B. Keller \& Moser 2013), die eine tiefere Zufriedenheit für Jugendliche mit sonderschulischem Hintergrund ausweisen. In der Studie von Keller und Moser (2013) beeinflusste v.a. ein fehlender Ausbildungsabschluss die Zufriedenheit negativ. Abweichungen von der Norm (sei es ohne Ausbildung oder arbeitslos zu sein) scheinen für die betroffenen Jugendlichen grundsätzlich eine Belastung zu sein. Ein Hinweis darauf sind auch die markant tieferen Zufriedenheitswerten von nicht beruflich integrierten Jugendlichen in unserer Studie.

Weiter zeigten unsere multivariaten Analysen, dass der sozioökonomische Status der Familie keinen Einfluss auf das Risiko der Erwerbslosigkeit nach Ausbildungsabschluss hat. Vermutlich hat dies damit zu tun, dass sich junge Erwachsene aus höheren sozialen Schichten eher eine Phase der Arbeitslosigkeit leisten können (Neuenschwander 2013, Erikson \& Jonsson, 1998). Aus diesem Grund und wegen höherer Bildungsaspirationen der Eltern (vgl. Neuenschwander et al., 2012) hätte man allerdings auch erwarten können, dass Lernende aus einer Familie mit höherem sozioökonomischen Status eher eine Weiterbildung in Angriff nehmen. Dies lässt sich durch unsere Analysen jedoch nicht bestätigen, wie auch der Migrationshintergrund keinen Einfluss auf diese beiden abhängigen Variablen hat. Dazu ist abschliessend zu bemerken, dass wir es bei der untersuchten 
Population mit Lernenden zu tun haben, die bis zum Befragungszeitpunkt am Ausbildungsende bereits einige Hürden überwunden haben und deshalb bezüglich Risikofaktoren homogener sind als z.B. zu Beginn der Ausbildung. Folglich könnten sich Effekte durch die soziale oder kulturelle Herkunft bereits in der Schulzeit, beim Eintritt oder während der Ausbildung ausgewirkt haben (vgl. Opheim, 2007) und so nur mittelbar relevant sein. Überraschend ist hingegen, dass die Ausbildungsbedingungen im Betrieb und in der Schule (Unterstützung, Vielseitigkeit) keinen oder nur einen marginalen Effekt haben. Das gleiche gilt für die persönlichen Dispositionen (Selbstwert), wobei einzig die Depressivität die Wahrscheinlichkeit, erwerbslos zu sein, etwas erhöht. Weiterführende Analysen der HfH-Längsschnittdaten (Hofmann et al., 2014) zeigen, dass es sich lohnt, das Wirkungsgefüge hier genauer zu untersuchen: So zeigt sich, dass die vermittelnden Pfade, über welche die soziale Unterstützung während der Ausbildung ihre Wirkung entfaltet, sich zwischen den beiden Lernorten unterscheiden: Betriebliche Berufsbildende beeinflussen via die Gestaltung der Aufgaben (Vielseitigkeit, Handlungsspielraum), in der Folge das Selbstwertgefühl der Lernenden, die karrierebezogenen Aspirationen und längerfristig die Wahrscheinlichkeit, eine Weiterbildung in Angriff zu nehmen. Die Berufsfachschullehrpersonen dagegen stärken die karrierebezogenen Aspirationen direkt und via das schulische Fähigkeitsselbstkonzept, welches sich dann weiter auf die Wahrscheinlichkeit sich weiterzubilden auswirkt.

Als Grenze der Studie ist zu erwähnen, dass ausschliesslich Lernende befragt wurden, die bis zum Ende der Ausbildung durchgehalten haben. Somit sind z.B. keine Aussagen zu Lehrabbrüchen und deren Hintergründen möglich und die Beurteilung der Ausbildung beruht auf einer selektiven Stichprobe. Weiter ist anzumerken, dass sich die Stichprobe über die drei Erhebungszeitpunkte hinweg um rund die Hälfte reduziert hat. Auch dieser Ausfall ist vermutlich selektiv. Allerdings zeigen zusätzliche Analysen, dass die Effekte sich nicht grundsätzlich verändern, wenn man mit verschiedenen Stichproben rechnet. Interessant wären zudem zusätzlich zu den subjektiven Einschätzungen objektive Daten zu den schulischen und betrieblichen Leistungen und verlässlichere Informationen dazu, ob die ehemaligen EBA-Lernenden nach Abschluss im ersten oder im zweiten Arbeitsmarkt angestellt sind.

Zusammenfassend ist festzuhalten, dass die EBA-Grundbildung v.a. für schulisch stärkere Lernende aus Sonderklassen oder -schulen eine Chance darstellt, insbesondere im Hinblick auf die spätere berufliche Integration. Handlungsbedarf besteht angesichts der relativ hohen Lehrabbruchquoten (Stern et. al. 2010) nach wie vor bei der begleitenden Unterstützung (,fachkundige individuelle Begleitung"), die sich idealerweise mit der schulischen und mit betrieblichen Situa- 
tion befassen sollte. Die festgestellten Branchenunterschiede sprechen ausserdem dafür, dass die Ausbildungssituation für jede Branche gezielt und unter Einbezug der betroffenen Branchenverbände analysiert werden sollte. Für Jugendliche, welche die Hürde EBA nicht schaffen, braucht es auf der anderen Seite Alternativen mit guten Anschlussoptionen, wie die Praktische Ausbildung nach INSOS (vgl. www.insos.ch). Eine zweite Hürde stellt der Übergang von der Ausbildung in den Arbeitsmarkt dar, die gemäss unseren Ergebnissen von Lernenden aus Sonderklassen/-schule etwas verzögert genommen wird. Diese Risiken könnten u.U. minimiert werden, wenn dieser Übergang bei diesen Jugendlichen zusätzlich begleitet würde, wie dies in Modellen von „supported employment“ bereits erfolgreich erprobt wird (vgl. Hofmann \& Schaub, 2014). Es wird weiter interessant sein zu beobachten, wie sich neue integrative Schulmodelle auf die weitere berufliche Laufbahn auswirken (Eckhart \& Sahli Lozano, 2014). Erste Ergebnisse zeigen, dass Jugendliche, die in einem integrativen Setting unterrichtet wurden, eher einen direkten Übertritt in die Ausbildung schaffen (Keller \& Moser, 2013). Wie (erfolgreich) sich ihre weitere berufliche Laufbahn gestaltet, hängt nicht zuletzt davon ab, ob und wie der integrative Grundgedanke auch in der Berufsbildung und in der Arbeitswelt etabliert und verankert werden kann.

\section{Literatur}

Baillod, J. (1992). Fluktuation bei Computerfachleuten: eine Längsschnittuntersuchung über die Beziehung zwischen Arbeitssituationen und Berufsverläufen. Frankfurt, Bern, New York, Paris, Wien: Peter Lang.

Bertschy, K., Böni, E., \& Meyer, T. (2007). An der zweiten Schwelle: Junge Menschen im Übergang zwischen Ausbildung und Arbeitsmarkt. Ergebnisübersicht des Jugendlängsschnitts TREE, Update 2007. Bern: TREE.

Eckhart, M., Blanc, P., \& Sahli, C. (2010). Ausbildungssituationen zwischen Wunsch und Wirklichkeit. Schweizerische Zeitschrift für Heilpädagogik, 16(3).

Eckhart, M., \& Sahli Lozano, C. (2014). Der lange Schatten der schulischen Separation: Ergebnisse einer Längsschnittuntersuchung. In M. P. Neuenschwander (Ed.), Selektion in Schule und Betrieb. Zürich/Chur: Rüegger Verlag.

Elfering, A., Semmer, N., Tschan, F., Kälin, W., \& Bucher, A. (2007). First years in job: A three-wave analysis of work experiences. Journal of Vocational Behavior, 70, 97-115.

Fasching, H. (2013). Interaktion von Behinderung und Geschlecht im Übergang von der Schule in Ausbildung und Beschäftigung. VHN (Vierteljahresschrift für Heilpädagogik und ihre Nachbargebiete), 82(1), 46-59.

Felkendorf, K., \& Lischer, E. (Eds.). (2005). Barrierefreie Übergänge? Jugendliche mit Behinderungen und Lernschwierigkeiten zwischen Schule und Berufsleben. Zürich: Verlag Pestalozzianum. 
Frese, M. (1999). Social Support as a moderator of the relationship between work stressors and psychological dysfuncioning: a longitudinal study with objective measures. Journal of Occupational Health Psychology, 4(3), 179-192.

Gaupp, N., Geier, B., \& Hupka-Brunner, S. (2012). Chancen bildungsbenachteiligter junger Erwachsener in der Schweiz und in Deutschland: Die (Nicht-)Bewältigung der zweiten Schwelle. Zeitschrift für Soziologie der Erziehung und Sozialisation, 32(3), 299-318.

Geier, B., Hupka-Brunner, S., \& Gaupp, N. (2013). Les trajectoires d'insertion des jeunes peu qualifiés en Suisse et en Allemagne. Chances of young adults from lower secondary schools with basic intellectual requirements in Switzerland and Germany: Mastering (or failing) the second threshold. Cahiers de la recherche sur l'éducation et les savoirs, 2013(4), 149-166.

Grob, A., \& Jaschinski, U. (2003). Erwachsen werden. Entwicklungspsychologie des Jugendalters (1. Auflage ed.). Weinheim, Basel, Berlin: Beltz Verlag.

Gyseler, D. (2008). Ausbildungswege von Lernenden mit besonderen Bedürfnissen. In K. Häfeli (Ed.), Berufliche Integration für Menschen mit Beeinträchtigungen - Luxus oder Notwendigkeit? (pp. 67-76). Luzern: Edition SH/CSPS.

Häfeli, K., \& Schellenberg, C. (2009). Erfolgsfaktoren in der Berufsbildung bei gefährdeten Jugendlichen. In EDK (Ed.), Studien + Berichte Bern: EDK Schweizerische Erziehungsdirektoren Konferenz.

Heinz, W. R. (2002). Transition discontinuities and biographical shaping of early work careers. Journal of Vocational Behavior, 60, 220-240.

Heslin, P. A. (2005). Conceptualizing and evaluating career success. Journal of Organizational Behavior, 26, 113-126.

Hofmann, C., \& Häfeli, K. (2010). Die Ausbildungssituation von Lernenden in einer Grundbildung mit eidgenössischem Berufsattest (EBA) in den Branchen Schreinerei und Hauswirtschaft : Zwischenbericht zur Erstbefragung der Lernenden am Ende der Ausbildung. Zürich: Interkantonale Hochschule für Heilpädagogik.

Hofmann, C., \& Häfeli, K. (2012). Subjektiver Laufbahnerfolg bei Leistungsschwächeren in einer Berufsbildung. Schweizerische Zeitschrift für Bildungswissenschaften, 1, 115-135.

Hofmann, C., \& Kammermann, M. (2008). Die zweijährige berufliche Grundbildung aus Sicht der Ausbildungsverantwortlichen in Berufsfachschule und Betrieb. Schweizerische Zeitschrift für Heilpädagogik, 14(6), 43-50.

Hofmann, C., \& Kammermann, M. (2009). Die zweijährige berufliche Grundbildung - ein Erfolgsmodell? Schweizerische Zeitschrift für Heilpädagogik 15(6), 27-34.

Hofmann, C., \& Schaub, S. (2014). „Supported education“ im ersten Arbeitsmarkt: Ausbildungssituation und berufliche Perspektiven. Schweizerische Zeitschrift für Heilpädagogik, 20, 25-32.

Hofmann, C., Stalder, B. E., Tschan, F., \& Häfeli, K. (2014). Support from teachers and trainers in Vocational education and training: The pathways to career aspirations and further career development. International Journal for Research in Vocational Education and Training, 1(1), 1-20.

Humphrey, S. E., Nahrgang, J. D., \& Morgenson, F. P. (2007). Integrating Motivational, Social, and Contextual Work Design Features: A Meta-Analytic Summary and Theoretical Extension of the Work Design Literature. Journal of Applied Psychology, 92(5), 1332-1356.

Hupka-Brunner, S., \& Wohlgemuth, K. (2014). Wie weiter nach der Schule? Zum Einfluss der Selektion in der Sekundarstufe I auf den weiteren Bildungsverlauf Schweizer Jugend- 
licher. In M. P. Neuenschwander (Ed.), Selektion in Schule und Arbeitsmarkt (pp. 99112). Zürich/Chur: Rüegger Verlag.

Hupka, S., Sacchi, S., \& Stalder, B. E. (2006). Does the Swiss VET System encourage inequity? Paper presented at the European Research Network on Transitions in Youth TIY, Marseilles.

Imdorf, C. (2014). Die Bedeutung von Schulqualifikationen, nationaler Herkunft und Geschlecht beim Übergang von der Schule in die betriebliche Berufsausbildung. In M. P. Neuenschwander (Ed.), Selektion in Schule und Arbeitsmarkt (pp. 41-62). Zürich/Chur: Rüegger Verlag.

Kammermann, M., Amos, J., Hofmann, C., \& Hättich, A. (2009). Integriert in den Arbeitsmarkt? Personen mit Berufsattest im Detailhandel und im Gastgewerbe ein Jahr nach Ausbildungsabschluss. Zürich: Hochschule für Heilpädagogik.

Kammermann, M., Hofmann, C., \& Hättich, A. (2009). Forschungsprojekt Arbeitsmarktfähigkeit und zweijährige berufliche Grundbildung. Skalendokumentation zur Ersterhebung bei den Lernenden. Zürich: Hochschule für Heilpädagogik.

Keller, F., \& Moser, U. (2013). Schullaufbahnen und Bildungserfolg. Auswirkungen von Schullaufbahnen und Schulsystem auf den Übertritt ins Berufsleben. Zürich/Chur: Rüegger Verlag.

Lehmann, R., \& Seeber, S. (2007). ULME III. Untersuchung von Leistungen, Motivation und Einstellungen der Schülerinnen und Schüler in den Abschlussklassen der Berufsschulen. Hamburg: Hamburger Institut für berufliche Bildung (HIBB).

Linnehan, F. (2003). A longitudinal study of work-based, adult-youth mentoring. Journal of Vocational Behavior, 63, 50-54.

Lischer, E. (2005). Berufliche Kurzausbildungen in der Schweiz: Die neue zweijährige Grundbildung mit Attest. In K. Felkendorff \& E. Lischer (Eds.), Barrierefreie Übergänge? (pp. 114-123). Zürich: Verlag Pestalozzianum.

Maaz, K., \& Neumann, M. (2014). Mechanismen schulischer Selektion. In M. P. Neuenschwander (Ed.), Selektion in Schule und Arbeitsmarkt. Zürich/Chur: Rüegger Verlag.

Moser, U. (2004). Jugendliche zwischen Schule und Berufsbildung. Bern: h.e.p. Verlag.

Müller, R. (2009). Berufswahl und Lehre. Berufliche Orientierungs- und Entscheidungsprozesse bei ausländischen und schweizerischen Jugendlichen (1. Auflage ed.). Bern: hep-Verlag ag.

Neuenschwander, M. P. (1998). Schule und Identität im Jugendalter I. Kurzdokumentation der Skalen und Stichproben (Forschungsbericht Nr. 18). Bern: Universität Bern, Abteilung Pädagogische Psychologie.

Neuenschwander, M. P. (2014). Selektion beim Übergang in die Sekundarstufe I und in den Arbeitsmarkt im Vergleich. In M. P. Neuenschwander (Ed.), Selektion in Schule und Arbeitsmarkt. Zürich/Chur: Rüegger Verlag.

Neuenschwander, M. P., Frey, M., \& Gasser, L. (2007). Übergang in die Sekundarstufe II und Wirkungen der Berufsbildung. Zürcher Ergänzungsstudie Berufsbildung - Schlussbericht. Zürich: Jacobs Center for Productive Youth Development, Universität Zürich.

Neuenschwander, M. P., Gerber, M., Frank, N., \& Rottermann, B. (Eds.). (2012). Schule und Beruf. Wege in die Erwerbstätigkeit. Wiesbaden: VS Verlag.

Neuenschwander, M. P., \& Grunder, H.-U. (Eds.). (2010). Schulübergang und Selektion. Zürich/Chur: Rüegger Verlag.

Ng, T. W. H., Eby, L. T., Sorensen, K. L., \& Feldman, D. C. (2005). Predictors of objective and subjective career success: A meta-analysis. Personnel Psychology, 58, 367-408. 
OECD. (2000). From Initial Education to Working Life. Making Transitions Work. Paris: OECD.

OECD. (2013). Education at a Glance 2013: OECD Indicators. Paris: OECD Publishing.

Opheim, V. (2007). Equal opportunities? The effect of social background on transition from education to work among graduates in Norway. Journal of Education and Work, 20(3), 255-282.

Pinquart, M., Juang, L., \& Silbereisen, R. K. (2003). Self-efficacy and successful school-towork transition: A longitudinal study. Journal of Vocational Behavior, 63, 329-346.

Prümper, J., Hartmannsgruber, K., \& Frese, M. (1995). KFZA. Kurz-Fragebogen zur Arbeitsanalyse. Zeitschrift für Arbeits- und Organisationspsychologie, 39(3), 125-131.

Raeder, S., \& Grote, G. (2003). Arbeitsmarktfähigkeit ersetzt Arbeitsplatzsicherheit. Die Volkswirtschaft, 13, 9-12.

Rosenberg, M. (1979). Conceiving the self. New York: Basic Books.

Rüfenacht, M., \& Neuenschwander, M. P. (2014). Jugendarbeitslosigkeit - Risikofaktoren und erfolgreicher Einstieg in die Erwerbstätigkeit. In M. P. Neuenschwander (Ed.), Selektion in Schule und Arbeitsmarkt. Zürich/Chur: Rüegger Verlag.

Scharenberg, K., Rudin, M., Müller, B., Meyer, T., \& Hupka-Brunner, S. (2014). Ausbildungsverläufte von der obligatorischen Schule ins junge Erwachsenenalter. Die ersten zehnt Jahre. Ergebnisübersicht der Schweizer Längsschnittstudie TREE, Teil I. Basel: TREE.

Schmid, E., \& Stalder, B. E. (2007). Lehrvertragsauflösung: direkter Wechsel und vorläufiger Ausstieg. Ergebnisse aus dem Projekt LEVA. Bern: Bildungsplanung und Evaluation der Erziehungsdirektion des Kantons Bern.

Schmid, E., \& Stalder, B. E. (2008). Lehrvertragsauflösung: Chancen und Risiken für den weiteren Ausbildungsweg. Ergebnisse aus dem Projekt LEVA (pp. 93). Bern: Bildungsplanung und Evaluation der Erziehungsdirektion des Kantons Bern.

Seibert, H., \& Solga, H. (2005). Gleiche Chancen dank einer abgeschlossenen Ausbildung? Zum Signalwert von Ausbildungsabschlüssen bei ausländischen und deutschen jungen Erwachsenen. Zeitschrift für Soziologie, 34(5), 364-382.

Semmer, N. K., Zapf, D., \& Dunckel, H. (1999). Instrument zur Stressbezogenen Tätigkeitsanalyse (ISTA). In H. Dunckel (Ed.), Handbuch psychologischer Arbeitsanalyseverfahren (pp. 179-205). Zürich: vdf: Hochschulverlag an der ETH.

Spiess Huldi, C., Häfeli, K., \& Rüesch, P. (2006). Risikofaktoren bei Jugendlichen und ihre Auswirkungen auf das Leben im Erwachsenenalter. Eine Sekundäranalyse der Zürcher Längsschnittstudie „Von der Schulzeit bis zum mittleren Erwachsenenalter“ (ZLSE). Luzern: Schweizerische Zentralsstelle für Heilpädagogik.

Stalder, B. E. (2012). School-to-work transitions in apprenticeship-based VET systems: the Swiss approach. In S. Billett, G. Johnson, S. Thomas, C. Sim, S. Hay \& J. Ryan (Eds.), The Experience of School Transitions: Policies, Practice and Participants (pp. 123-139). Berlin: Springer.

Stalder, B. E., Meyer, T., \& Hupka-Brunner, S. (2008). Leistungsschwach - Bildungsarm? Ergebnisse der TREE-Studie zu den PISA-Kompetenzen als Prädiktoren für Bildungschancen in der Sekundarstufe II. Die Deutsche Schule, 100(4), 436-448.

Stalder, B. E., Meyer, T., \& Hupka-Brunner, S. (2011). TREE Project Documentation. In M. M. Bergman, S. Hupka-Brunner, A. Keller, T. Meyer \& B. E. Stalder (Eds.), Youth Transitions in Switzerland: Results from the TREE Panel Study (pp. 66-87). Zürich: Seismo. 
Stalder, B. E., \& Schmid, E. (2006). Lehrvertragsauflösungen, ihre Ursachen und Konsequenzen. Ergebnisse aus dem Projekt LEVA. Bern: Erziehungsdirektion des Kantons Bern (Bildungsplanung und Evaluation).

Stern, S., Marti, C., von Stokar, T., \& Ehrler, J. (2010). Evaluation der zweijährigen beruflichen Grundbildung mit EBA. Schlussbericht. Zürich/Lausanne: INFRAS/Idheap.

Venetz, M., Tarnutzer, R., Zurbriggen, C., \& Sempert, W. (2012). Emotionales Erleben im Unterricht und schulbezogene Selbstbilder. Vergleichende Analysen von Lernenden in integrativen und separativen Schulformen. Bern Edition SZH/CSPS.

Watermann, R. (2000). Berufliche Wertorientierungen im Wandel. Eine Kohortenanalyse zur Dynamik arbeitsbezogener Einstellungen anhand von ALLBUS-Umfragedaten. Münster: Institut für sozialwissenschaftliche Forschung e.V.

Dieses Buch wird unter der Creative Commons Namensnennung-Nicht kommerziell 4.0 International Lizenz (http://creativecommons.org/licenses/by-nc/4.0/deed.de) veröffentlicht, welche für nicht kommerzielle Zwecke die Nutzung, Vervielfältigung, Bearbeitung, Verbreitung und Wiedergabe in jeglichem Medium und Format erlaubt, sofern Sie den/die ursprünglichen Autor(en) und die Quelle ordnungsgemäß nennen, einen Link zur Creative Commons Lizenz beifügen und angegeben, ob Änderungen vorgenommen wurden. 